\title{
Study on the Effects of Long-Term Aerobic Exercise Combined with Resistance Training on Cardiac Function, Exercise Tolerance and Quality of Life in Patients with Myocardial Ischemia caused by Coronary Artery Disease
}

\author{
Yuan Zhang \\ Northwest University, Xi'an, Shanxi, 710069
}

\begin{abstract}
Coronary heart disease caused by myocardial ischemia is a common clinical heart disease. As coronary heart disease in patients with coronary atherosclerosis, arterial stenosis and resulting in insufficient blood flow to the coronary artery, myocardial blood flow decreased. The main clinical symptoms of chest pain, shortness of breath, palpitations, chest tightness, fatigue, etc., a serious threat to the lives and safety of patients with quality of life. With the aerobic exercise and resistance exercise in clinical applications, and gradually developed to improve coronary heart disease caused by myocardial ischemia in patients with cardiac function and exercise tolerance and quality of life effective means.
\end{abstract}

Keywords: CHD, Aerobic Exercise, Resistance Training, Recovery

\section{Introduction}

With the development of modern medical technology, especially the extensive use of percutaneous coronary artery stenting (PCI) and coronary artery bypass grafting $(\mathrm{CABG})$, coronary heart disease (CHD) is a common disease and frequently-occurring disease threatening human health. Mortality in acute coronary events is greatly reduced. However, long-term, CHD patients with recurrence rate and mortality is still high. In recent years, people gradually 
recognized that cardiac rehabilitation is the treatment of stable CHD important and effective way. Exercise training is the core of cardiac rehabilitation, but the determination of exercise intensity is still controversial, high intensity intermittent training is considered to be more effective exercise training methods, which is characterized by intensity, time is short, intermittent, effective, side effects Small, easy to adhere to, but because there is a certain risk, there is a big controversy. Therefore, this study intends to use high-intensity and low-intensity aerobic training methods to observe its impact on exercise tolerance in patients with CHD, for large-scale clinical application of the lay basis.

\section{Resistance Training and Cardiac Rehabilitation Training}

Resistance training, including lifting dumbbells, sit-ups and other muscle training, also known as muscle strength training. Jogging, climbing, climbing, swimming, boating, ball, running and other aerobic exercise can improve heart and lung function and enhance resistance; and lifting dumbbells, weightlifting, stretch belt, fitness training, such as resistance training muscle, muscle force. Diabetic patients in the aerobic exercise will consume energy, this will reduce blood sugar. Therefore, in the training process, aerobic exercise should be the "main force." Compared to aerobic exercise, resistance training can better optimize and enhance the antioxidant capacity of skeletal muscle in diabetic patients, can help maintain a hypoxic state, which can promote insulin resistance in patients with reduced. Therefore, the two exercise can be complementary. Patients with coronary heart disease early and early best combination of aerobic exercise resistance training exercise, resistance training should be less than aerobic exercise. How to allocate the proportion of the two exercise methods, but also need to see the specific circumstances of patients. Determine the appropriate amount of exercise

Cardiac rehabilitation focuses on exercise training, and how to determine exercise intensity is a complex problem. Excessive exercise intensity of the patients have a certain risk, and too low exercise intensity will cause the effect of rehabilitation is poor. In recent years, high-intensity cardiac rehabilitation exercise has been involved in foreign countries, the risk has been a better assessment and control, but still lack the necessary clinical experience in our country. The so-called high-intensity training: power in the incremental exercise evaluation test, the test subjects due to exhaustion or other symptoms and termination of the test when the power of the maximum power, is generally considered to take the maximum power of $60 \%$ to $80 \%$ can be considered high strength. Low intensity is not exactly defined, generally take the maximum power of $30 \%$ to $60 \%$ as low intensity. 


\section{Research Data and Methods}

From October 2010 to September 2011, 60 patients with CHD, including 35 males and 25 females, aged from 65 to 75 years (mean 71.6 years) were recruited in our hospital. The diagnostic criteria were based on the Chinese Medical Association's diagnosis and treatment of coronary heart disease Guidelines, and to determine the complications without prejudice to the implementation of cardiac rehabilitation. Sixty patients were randomly divided into three groups: high-intensity aerobic training group ( $70 \%$ of peak power) in 20 cases, including 11 males and 9 females, the average age (72.7) years, 20 cases of low-intensity aerobic training group ( $70 \%$ of the peak power), including 12 males and 8 females, the average age (mean age, mean heart function, (70.5 years), 15 cases of cardiac function grade, 5 cases of Ò grade, 16 cases of angina pectoris, 4 cases of old myocardial infarction, 20 cases of control group, including 12 males and 8 females, mean age (73.8) Functional $\tilde{\mathrm{N}}$ grade 14 cases, Ò grade 6 cases, angina pectoris in 14 cases, old myocardial infarction in 6 cases. There was no statistical difference between the three groups $(\mathrm{P}>0.05)$.

The patients in the control group received $50 \%$ of the peak power as the training intensity, and continued training for $20 \mathrm{~min} /$ time. There were 5 patients in the low-intensity continuous training group and 5 in the control group before and after the training, and the control group was given health education, drug therapy and maintaining the daily life. Min for 3 weeks and 8 weeks for highintensity intermittent training group, $70 \%$ for high-intensity intermittent training group as high-intensity training intensity, 4 for each training, $5 \mathrm{~min}$ for each group, and 5 for rest Min, the total time of $20 \mathrm{~min}, 3$ times / week, a total of 8 weeks, before and after training have 5 minutes of preparatory activities and relaxation activities. The training intensity can be based on individual patient tolerance, as appropriate, increase or decrease of $5 \%$ to $10 \%$, and as far as possible in the air fresh place. Low intensity and high intensity group 2 during exercise ECG monitoring, no significant symptoms of angina pectoris and electrocardiogram significantly ST-T changes.

All patients underwent cardiopulmonary exercise test and cardiac ultrasound examination before and after training. Cardiac ultrasonography was performed with PHILIPS Color Doppler Ultrasound System. The patients in the exercise training group were examined by echocardiography before and 8 weeks after the training. The cardiac echocardiography was also performed in the control group. The left ventricular ejection fraction Cardiopulmonary exercise test was used to test the exercise duration, peak power, oxygen pulse and anaerobic threshold (AT) in $\mathrm{K} 4 \mathrm{~b} 2$ cardiopulmonary exercise test training system.

\section{Resistance Training and Aerobic Exercise}

$(1 \mathrm{RM})$ and the maximal load (10RM) of the isometric contraction of the muscles to be trained $(10 \mathrm{RM})$ were taken as the reference, and the exercise program was 
developed according to the different physical conditions of the patients before the resistance training. The main training site for the lower back, abdominal muscles and upper and lower limb muscle group. Training intensity Exercise intensity for the first week after surgery, family support in bed without load-bearing quadriceps and other contractions. Muscle tension after $10 \mathrm{~s}$ relaxation of $15 \mathrm{~s}$ for 1 time, 200 times a day. From the 2 nd to 5 th week after the operation, the patients were assisted with or assisted with wall motion, and the hip joints were flexed for $15 \mathrm{~min}, 10$ times per day, and the amplitude and time were gradually increased. Week, on the basis of the above-mentioned training, increase the chest push exercise, shoulders, triceps extension, biceps flexion four movements, each prescribed action for 3 seconds or more, each movement $20 \mathrm{~min}, 3$ times a day. The main forms of training are sandbags, dumbbell elastic band and weight training facilities.

Aerobic training: the patient's maximum oxygen consumption and anaerobic threshold assessment to determine the exercise intensity. In the first week after operation, the leg lift was carried out, and the limbs were gradually lifted until the thighs were removed from the bed. After 10 seconds of rest, each group was given 10 times, 5 times a day, gradually increasing the distance from the bed. 2 to 5 weeks, bedside sitting training and knee straightening exercise. Gradually increase exercise intensity. 6 to 12 weeks after the normal walking, each walking 10 min, 4 times a day, pay attention to adjust the walking stride and moving speed, gradually increase the number of walking and extended walking time, gradually increased from $100 \mathrm{~m}$ to $500 \mathrm{~m}$.

\section{Results Analysis}

Cardiac ultrasonography showed no significant difference in LVEF between groups before rehabilitation training $(\mathrm{P}>0.05)$. After 8 weeks of rehabilitation training, LVEF of patients with low-intensity and high-intensity aerobic training group improved, but not with control group Sex difference $(\mathrm{P}>0.05)$.

There was no significant difference in the duration of test, peak power, oxygen pulse and anaerobic threshold between the three groups before treatment $(\mathrm{P}>0.05)$, the difference after rehabilitation training (before training - before training) ( $\mathrm{P}<0.05$ or $\mathrm{P}<0.01)$. AT was only higher in the high intensity group than in the control group $(\mathrm{P}<0.01)$, and the difference was statistically significant $(\mathrm{P}<0.05)$.

In this study, after 8 weeks of rehabilitation exercise training, low-intensity and high-intensity aerobic training group LVEF patients increased, but no significant difference with the control group. It is suggested that aerobic exercise can improve the cardiac function of patients with stable CHD, but the effect is not great, and can not improve the heart function of patients, which is not consistent with the results of some domestic researchers. From the aspect of exercise endurance of patients with CHD, whether low-intensity continuous aerobic training or high-intensity intermittent aerobic training to improve exercise tolerance in patients with $\mathrm{CHD}$ are helpful, the aerobic exercise time, peak power, 
$(\mathrm{P}<0.05)$, and the oxygen pulse was significantly increased $(\mathrm{P}<0.05)$. Especially for high-intensity trainer, its aerobic exercise capacity increased more significantly, anaerobic threshold is also significantly increased, suggesting that high-intensity aerobic exercise for CHD patients exercise endurance to enhance more effective. In addition, Inga et al. Compared high-intensity (85\% to 95\% of heart rate to maximal heart rate reserve) with moderate intensity (60-70\% of maximal heart rate reserve) and found that high intensity improved endothelial function, Reduce the incidence of cardiovascular events in obese people. 24 subjects were randomly assigned to high-intensity group (in the heart rate of anaerobic threshold) and low-intensity group (to reach maximum heart rate reserve $50 \%$ of the heart rate), with a fixed bike for 4 weeks, 5 times / Week, 45 min / training. Compared with the low intensity group, the maximal exercise oxygen uptake and the maximum ventilation decreased in the high intensity training group, which were consistent with the results of this study. But also fully demonstrated the benefits of high-intensity aerobic exercise for patients with CHD.

\section{Conclusion}

In summary, high-intensity intermittent aerobic training, which mainly consists of lower limb movements, can better improve the exercise tolerance of patients with stable CHD, the effect is significantly higher than low-intensity aerobic endurance training, and spend a shorter time, The patient's compliance has been greatly improved. Based on this, high-intensity aerobic training should be an important choice in cardio rehabilitation cardio training, its clinical application will effectively improve the efficiency of cardiac rehabilitation.

\section{References}

[1] Wang Zhen. Progress in the etiology of chronic heart failure [J]. Hebei Medical Journal. 2011 (14)

Effect of aerobic exercise on skeletal muscle function in patients with chronic heart failure [J] .Hebei Medicine and Materia Medica, 2010, (6): 124-12.

[2] Ren Li, Gao Qianjin, Zhang Yanxia,

[3] Ren Li, Zhang Yanxia, Li Xiaolin, Gao Qianjin. Effects of aerobic exercise on myocardial injury and plasma CGRP and ET-1 in patients with chronic heart failure [J]. Hebei Medicine and Pharmacy,

[4] Yu Ruiying, Wang Jiying, Jiang Hongyan, Li Li, Li Ruijie.Effect of rehabilitation exercise on quality of life in elderly patients with chronic heart failure [J]. Chinese Journal of Cardiovascular Rehabilitation Medicine.

[5] chronic heart failure diagnosis and treatment guidelines [J].Chinese Journal of cardiovascular disease. 2007 (12) 